\title{
Affective Computing Combined with Android Science
}

\author{
Christian Becker-Asano
}

Published online: 25 May 2011

(C) Springer-Verlag 2011

\begin{abstract}
In this report a number of research projects are summarized that aimed at investigating the emotional effects of android robots. In particular, those robots are focused on that have been developed and are incessantly being improved by Hiroshi Ishiguro at both the Advanced Telecommunications Research Institute International (ATR) in Kyoto and Osaka University in Osaka, Japan. Parts of the reported empirical research have been conducted by the author himself during a two-year research stay at ATR as post-doctoral fellow of the Japan Society for the Promotion of Science.

In conclusion, Affective Computing research is taken to the next level by employing physical androids rather than purely virtual humans, and Android Science benefits from the experience of the Affective Computing community in devising means to assess and evaluate a human observer's subjective impressions that android robots give rise to.
\end{abstract}

Keywords Affective computing - Android science · Robotics

\section{Introduction}

This project report presents an overview of the efforts to apply Affective Computing methodology to Android Science. We will concentrate on the android robots of the "Repliee" and "Geminoid" series, which are developed and incessantly

I would like to thank my former host Prof. Hiroshi Ishiguro for his generous support during the two years of collaborative research at ATR, Japan.

\section{Becker-Asano ( $\square)$}

Freiburg Institute for Advanced Studies (FRIAS),

Interdisciplinary Research Group, Albert-Ludwigs-Universität

Freiburg, Albertstraße 19, 79104 Freiburg, Germany

e-mail: christian@becker-asano.de being improved in the Kansai area of Japan, namely in Kyoto and Osaka.

Affective Computing and Android Science, two rather young sub-disciplines of applied computer science, share a common interest in that researchers of both faculties try to gain a deeper understanding of inherently human phenomena. "Affective Computing" [19] is concerned with enabling machines to recognize, express, model, communicate, and respond to emotion. In doing so, their proponents also aim to understand the fuzzy concept of "affect", which subsumes such mental phenomena as personality, mood, and emotion. Therefore, most researchers following the Affective Computing paradigm are naturally involved in interdisciplinary projects comprising of cognitive scientists, psychologists, philosophers, and the like. Similarly, "Android Science" research is characterized as a "cross-interdisciplinary framework" [11] that bridges robotics and cognitive science. Proponents of this approach argue that humanhuman interaction can be studied systematically making use of android robots. Indeed, their high degree of anthropomorphism makes them indistinguishable from humans at first sight. This motivation easily connects with the motives behind Affective Computing, because the slightest irregularities of an android's facial movements often lead to strong emotional reactions (the so-called "uncanny feelings") in the observers.

The remainder of this paper is structured as follows: In the following section the general motivation behind applying Affective Computing methodology to Android Science is detailed further. In Sect. 3 a selection of research projects is introduced that involve androids of different types. Accordingly, their results and conclusions are summarized in light of the research goals pursued by the Affective Computing community, which gives rise to a number of challenging research questions. Finally, in Sect. 4 general conclusions 
are drawn and it is argued that both faculties, Affective Computing and Android Science, will benefit from a continued cooperation.

\section{Motivation}

The term "Affective Computing" has been criticized for being inadequate or even misleading [9]. Of course, computing itself can hardly be "affective" in the sense of being emotional, but as clarified by Hudlicka [10] the aim of Affective Computing should be to construct machines that are "capable of recognizing and 'simulating' affect" to allow the researcher to "better understand the capabilities (and limitations) of our affective-cognitive system [..]." Thus, it is not taken for granted that the inclusion of affect simulation and/or recognition capabilities will necessarily improve Human-Computer Interaction.

Furthermore, it has been critically noted that a computers lack of a physical body might impede all attempts to model its emotional expressions appropriately [18]. In 2003 the assessment that "existing attempts at expressing emotions in robots [..] are unrealistic and therefore unconvincing" $[18$, p. 61] might have been correct, but with the advanced design of android robots in recent years the situation has changed dramatically. In the beginning of the new millennium most Affective Computing researchers interested in deep models of affect and its role in social interaction concentrated on Virtual Reality scenarios, in which Virtual Humans [5] serve as testbeds. The notable exceptions have designed rather animal- or cartoon-like robotic systems (e.g., $[1,7])$ thereby successfully avoiding to give rise to uncanny feelings in the observer. Unfortunately, the findings achieved with less anthropomorphic robots are of limited help in explaining emotion-related phenomena of humanhuman interaction.

With android robots such as "Barthoc" [22], "Repliee Q2" [13], or "Geminoid HI-1" [17], which feature a very high degree of anthropomorphism, researchers can now investigate these emotion-related phenomena in such ways that their results can be relevant to the neighboring disciplines as well. Designing and programming such highly anthropomorphic robots, however, is known to be very challenging, because the slightest inaccuracies can give rise to negative feelings in human observers. This "uncanny feeling" [12] is related to the hypothesis of an "uncanny valley" [14], which is a central topic of investigation in Android Science $[6,12]$.

\section{Research Projects with Android Robots}

\subsection{Repliee Q2}

The android Repliee Q2 (cf. Fig. 1) is an upgraded version of Repliee Q1, which had been presented to the public at

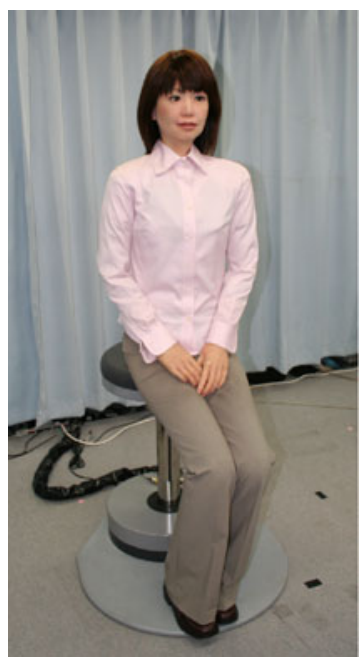

(a) Repliee Q2 installed at Osaka University, Japan

Fig. 1 The android robot Repliee Q2 (credit: Prof. Ishiguro at Osaka University and Kokoro Co., Ltd.)

the 2005 World Expo in Aichi, Japan. With 13 degrees of freedom in its head, three in its neck, nine in each of the arms, two in each hand, and four in its torso, the Q2 version consists of 42 degrees of freedom in total as compared to 31 degrees of freedom of the Q1 version [13]. Although a sensor network enables Repliee Q2 to react on the presence of visitors autonomously [8], it is as often tele-operated from a remote location.

Its human-likeness has been studied extensively by evaluating eye gaze patterns of human interactands during faceto-face conversations [21]. These experiments confirmed the importance of appropriate, high quality head and body movements, if the android is to be treated similarly to a human. During several clinical examination interviews, involving a medical doctor and a patient, Repliee Q2 has been employed as a passive bystander [23]. The question was whether or not the sheer presence of the android would already have a similar effect as the presence of a human nurse. ${ }^{1}$ After the examinations the patients were asked six questions that referred to their impressions of the doctor's behavior and attitude only. Nonetheless, significant differences were found depending on both the presence or absence of Repliee Q2 and whether or not it was smiling and nodding. A non-responsive android had the highest negative influence on the evaluations of the doctor's performance, such that in this case it seems better to completely remove the android from the examination room. Only if the android performed situation-appropriate smiling and nodding, it had a positive influence on the patients' evaluations of the doctor.

\footnotetext{
${ }^{1}$ In Japanese hospitals it is very common that a nurse stands behind the doctor during clinical examination interviews such as the ones targeted here.
} 

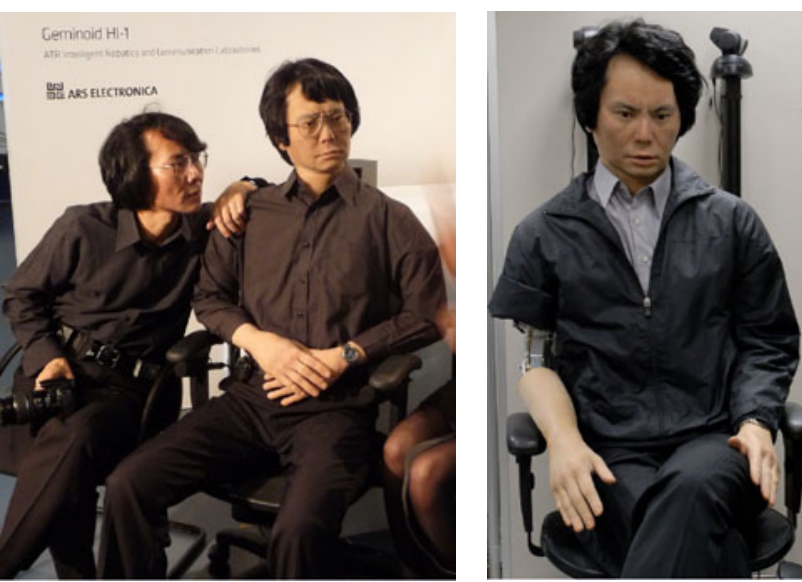

(a) Hiroshi Ishiguro (left) with his (b) Geminoid HI-1 with android Geminoid HI-1 at ARS its right arm uncovered Electronica festival 2009

Fig. 2 The android robot Geminoid HI-1

The android's facial expressions are rather limited, because its initial facial expression was modeled to feature a light smile. Accordingly, Repliee Q2 cannot frown or perform a 'sad' facial expression with both corners of its mouth pointing down. These inefficiencies have been addressed with the invention of "Geminoid F", which will be introduced in Sect. 3.3.

\subsection{Geminoid HI-1}

The development of the android robot "Geminoid HI-1" [17] was finished in 2006 and it is the first of a series of Geminoid-type androids. The term "Geminoid" is derived from the Latin word "geminus" meaning twin and the postfix " oides" meaning similarity. In fact, a Geminoid-type android is built to match a real person's outer appearance as closely as possible and Geminoid HI-1 (cp. Fig. 2) $)^{2}$ is supposed to be the robotic twin of Hiroshi Ishiguro. With such Geminoids his team tries to investigate the essential components of human-human multimodal interaction that let us believe that the other is indeed human. As current AI technology and our understanding of the processes behind multimodal interaction are both still too limited to achieve completely autonomous human-like behavior of Geminoidtype androids, these robots are mainly tele-operated during interaction. Only eye blinking and breathing are driven autonomously and their animation is combined with teleoperation control on the fly.

Geminoid HI-1 is driven by a total of 50 mostly pneumatic actuators enabling it to raise its arms, perform a point-

\footnotetext{
${ }^{2}$ Credit for Figs. 2, 3, and 4: Dr. Ishiguro is ATR Fellow and Professor of Osaka University. The Geminoid has been developed in ATR's Intelligent Robotics and Communication laboratories.
}

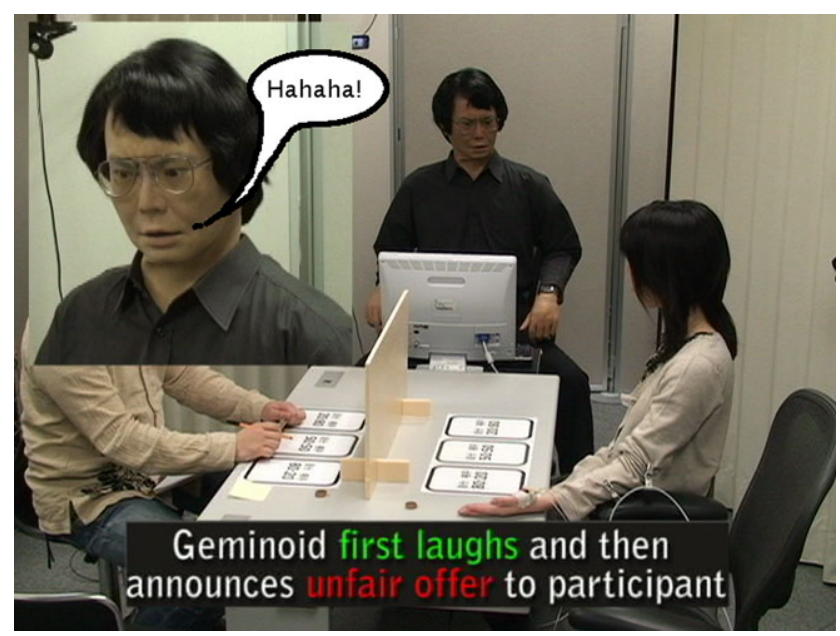

Fig. 3 Geminoid HI-1 laughing at the participant [2]

ing gesture with its index finger of the right hand, grab something with its left hand, tap with its feet independently of each other, and — last but not least—move its head and perform a number of facial expressions. Its limited facial expressivity, however, does not allow for detailed studies on the interpersonal effects of this android's emotional expressions. The actuators in the face are mainly used to let it perform facial movements of its mouth in synchrony with the tele-operator's voice; cf. [6, 17] for further details on the tele-operation.

Laboratory Studies From 2006 until Summer 2009 empirical research with Geminoid HI-1 was confined to taking place in ATR's Intelligent Robots and Communications laboratories. The role of the android's eye gaze in face-to-face interaction [15] as well as its power to transmit a remote person's "presence" [16] have been investigated empirically.

As Geminoid HI-1's facial expressivity is rather limited, we decided to start testing other means of emotion expression, namely laughter [2]. Based on the experience gained with two humanoid robots of the Robovie series [4] we chose three instances of recorded, male, human laughter and designed appropriate bodily and facial animations for Geminoid HI-1. These were applied to a playful interaction, in which a confederate student of the lab played a variant of the Ultimatum Game against a total of 36 invited participants; cf. Fig. 3. Geminoid HI-1-in the role of a supposedly neutral experimenter-started laughing whenever our student made an unfair offer.

After each game session the participants were asked to report on their feelings by means of a translated version of the Geneva Emotion Wheel [2]. A first analysis of the collected data suggests that Geminoid HI-1's laughter was interpreted rather negatively as "laughing at" the participant [2]. A more detailed analysis together with results of a recently conducted follow-up study, however, suggest mostly positive 


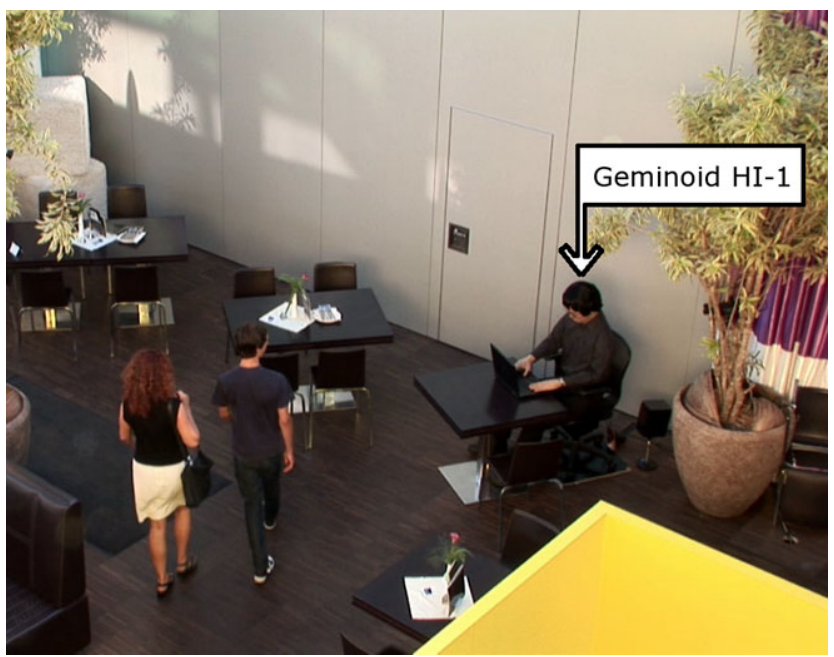

Fig. 4 Geminoid HI-1 secretly installed in "Café CUBUS" at ARS Electronica Center in Linz, Austria

effects of the same multimodal laughter. In general, dealing with highly subjective phenomena such as "emotions" together with a purely empirical research methodology can become as challenging as it is daunting at times and further experiments are needed to clarify the emotional effects of android laughter.

In the Wild In Summer 2009 Geminoid HI-1 was installed for the first time in a public location. During August it was seated behind a table in a public café located on the top floor of the ARS Electronica Center in Linz, Austria; cf. Fig. 4. During the opening times of the café we collected 20 days of non-stop video recordings from five different cameras in parallel to analyze, under which conditions Geminoid HI-1 was (1) recognized at all, (2) mistaken for a real human, or (3) identified as an android robot. Geminoid HI-1's behavior was systematically modified from only performing idle movements whilst looking down at its laptop, over automatically looking back at visitors whenever those looked straight into the camera behind it, to fully tele-operated and responsive to the visitors' addressing it. Ninety-one randomly selected visitors had been interviewed after they had walked by Geminoid HI-1's location.

A first analysis of the data [20] revealed that only 43 ( $\sim 47 \%$ ) of the interviewed visitors identified Geminoid HI1 as an android robot and 18 visitors $(\sim 20 \%)$ mistook it for a real (human) man, esp. when Geminoid was in the completely non-interactive mode. When Geminoid HI-1 returned a visitor's gaze, he or she was not only looking longer at it, but was also more likely to detect its robotlikeness. Interestingly, however, only three visitors reported unrequested that Geminoid HI-1 had given them an uneasy feeling.

In September 2009 Geminoid HI-1 was moved to the basement of the ARS Electronica Center to be exhibited
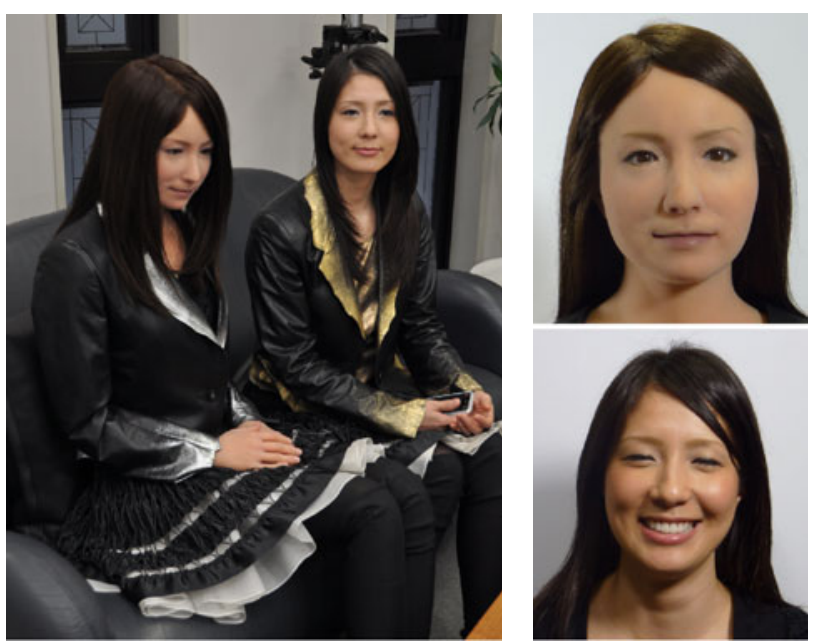

(a) Geminoid F (left) and its model (b) A smiling Gemiperson (right) noid F (top) and its model person (bottom)

Fig. 5 The android robot Geminoid F developed by Osaka University and ATR

as a festival attraction; cf. Fig. 2(a). Visitors of the festival could not only sit down next to the tele-operated Geminoid HI-1, but they could also try to tele-operate it themselves. Twenty-four structured interviews with randomly selected visitors, who had interacted with Geminoid HI-1 just before, revealed that approx. 37\% felt uneasy and another approx. $29 \%$ even enjoyed the interaction [6]. With respect to the visitors' feelings we found that fear was predominantan emotion that often results from a person's inability to predict future events. This effect was most likely caused by the operator's difficulty to direct Geminoid HI-1's gaze appropriately together with Geminoid HI-1's general inability to produce situation-appropriate social cues such as a smile or laughter.

Taking these experiences into account a new Geminoid has recently been developed focusing on facial expressivity, namely Geminoid F.

\subsection{Geminoid F}

As was the case for Geminoid HI-1, the android robot Geminoid $\mathrm{F}$ has also been modeled after a real person; cf. Fig. 5(a). Its agility, however, is much more limited than that of Geminoid HI-1, because it lacks actuators in its limbs such that it cannot move its arms, hands, or legs. One of its twelve actuators is used to move its body (front and back) and all remaining eleven actuators are responsible for head and facial movements (cf. [3] for details).

It was explicitly designed not only to be more affordable and easier to install than Geminoid HI-1, but also to feature much more natural facial expressions. Indeed, results of an 
empirical online survey [3] indicate that Geminoid F's facial portray of most basic emotions can be reliably identified by human observers with recognition rates ranging from 54.5\% for an angry expression up to 78.5\% in case of a happy expression (cp. Fig. 5(b)). Portraying fear, however, seems to be impossible with the current design of Geminoid F, because its recognition rate remained below level of chance. ${ }^{3}$ Geminoid F's mechanical inability to furrow its brows might impede all attempts to model a convincing fearful facial expression, but even for the model person portraying a fearful expression has proven to be most difficult with a recognition rate of $61.5 \%$ [3].

\subsection{Discussion}

All the above mentioned research projects give rise to a number of challenging new research questions. Which factors are most relevant to diminish the uncanny feeling that observers get: An android's gender, application context, multimodal interactivity, facial expressiveness, or something else? How do these factors combine? For example, in which situational contexts are facial expressions more important than communicational abilities? How can these multimodal abilities be driven autonomously and in concert, such that convincing and trustworthy artificial companions are achieved? Which ethical and judicial aspects need to be addressed concerning the use of such new types of robots?

Clearly, working on these questions affords continuous exchange with a number of neighboring disciplines, making this endeavor a prime example of interdisciplinary research.

\section{Conclusions and Outlook}

This article explained the motivation behind combining Affective Computing with Android Science.

On the one hand, for the Affective Computing community an android's sophisticated design allows to take empirical research on the effects of emotional expressions to the next level, because these highly anthropomorphic robots share the same physical space with us-in contrast to their similarly anthropomorphic virtual counterparts. In other words, people are likely to be less afraid of an angry virtual human than of a similarly expressive, angry android, because the latter could be expected to physically harm the human observer.

On the other hand, the methodologies devised and tested by the Affective Computing community have proven useful in assessing the subjective impressions that android robots

\footnotetext{
${ }^{3}$ As for each of the presented six facial expressions seven labels could be chosen independently, in one out of seven cases (14.2\%), a "correct" label could have been assigned by chance.
}

give rise to. The derived conclusions, in turn, aid the continuous improvement of android design letting them become even better tools for research on fundamental questions regarding human presence and multi-modal social interaction.

Finally, I hope that the broader Artificial Intelligence community agrees on the usefulness of these androids to work empirically on such general questions as which types of embodiment are best suited to achieve artificial agents that are not only rationally, but also socially intelligent.

Acknowledgements This work was partially supported by Grant-in Aid for Scientific Research (S), KAKENHI (20220002), by a postdoctoral fellowship of the Japan Society for the Promotion of Science and by a return fellowship of the Alexander von Humboldt-foundation.

\section{References}

1. Bartneck C (2003) Interacting with an embodied emotional character. In: Intl conf on designing pleasurable products and interfaces, DPPI'03. ACM, New York, pp 55-60

2. Becker-Asano C, Ishiguro H (2009) Laughter in Social Robotics-no laughing matter. In: Intl workshop on social intelligence design (SID2009), pp 287-300

3. Becker-Asano C, Ishiguro H (2011) Evaluating facial displays of emotion for the android robot Geminoid F. In: Workshop on affective computational intelligence, IEEE 2011 symposium series on computational intelligence, pp 22-29

4. Becker-Asano C, Kanda T, Ishi C, Ishiguro H (2010) Studying laughter combined with two humanoid robots. AI \& Society. Online first

5. Becker-Asano C, Kopp S, Pfeiffer-Leßmann N, Wachsmuth I (2008) Virtual humans growing up: from primary toward secondary emotions. KI Zeitschift (German Journal of Artificial Intelligence) 1:23-27

6. Becker-Asano C, Ogawa K, Nishio S, Ishiguro H (2010) Exploring the uncanny valley with Geminoid HI-1 in a real-world application. In: IADIS intl conf interfaces and human computer interaction, pp 121-128

7. Breazeal C (2003) Emotion and sociable humanoid robots. Int J Human-Comput Stud 59:119-155

8. Chikaraishi T, Minato T, Ishiguro H (2008) Development of an android system integrated with sensor networks. In: IEEE/RSJ intl conf on intelligent robots and systems, pp 326-333

9. Hollnagel E (2003) Is affective computing an oxymoron? Int J Human-Comput Stud 59:65-70

10. Hudlicka E (2003) Response: is affective computing an oxymoron? Int J Human-Comput Stud 59:71-75

11. Ishiguro $H$ (2005) Android science: toward a new crossinterdisciplinary framework. In: CogSci 2005 workshop "Toward social mechanisms of android science", pp 1-6

12. MacDorman KF, Ishiguro $H$ (2006) The uncanny advantage of using androids in cognitive and social science research. Interact Stud 7(3):297-337

13. Minato T, Shimada M, Itakura S, Lee K, Ishiguro H (2005) Does gaze reveal the human likeness of an android. In: Intl conf on development and learning, pp 106-111

14. Mori M (1970) Bukimi no tani [the uncanny valley]. Energy 7(4):33-35

15. Mutlu B, Yamaoka F, Kanda T, Ishiguro H, Hagita N (2009) Nonverbal leakage in robots: communication of intentions through seemingly unintentional behavior. In: ACM/IEEE intl conf on human robot interaction, pp 69-76 
16. Nishio S, Ishiguro H, Hagita N (2007) Can a teleoperated android represent personal presence? - a case study with children. Psychologia 50(4):330-342

17. Nishio S, Ishiguro H, Hagita N (2007) Geminoid: teleoperated android of an existing person. In: de Pina Filho AC (ed) Humanoid robots, new developments. Springer, Berlin, pp 343-352

18. Picard R (2003) Affective computing: challenges. Int J HumanComput Stud 59:55-64

19. Picard RW (1997) Affective computing. MIT Press, Cambridge

20. von der Pütten AM, Krämer NC, Becker-Asano C, Ishiguro H (2011) An android in the field. In: ACM/IEEE intl conf on humanrobot interaction, Lausanne, Switzerland, pp 283-284

21. Shimada M, Ishiguro H (2008) Motion behavior and its influence on human-likeness in an android robot. In: Proc of the annual meeting of the cognitive science society, pp 2468-2473

22. Spexard T, Hanheide M, Sagerer G (2007) Human-oriented interaction with an anthropomorphic robot. IEEE Trans Robot 23(5):852-862

23. Takano E, Chikaraishi T, Matsumoto Y, Nakamura Y, Ishiguro H, Sugamoto K (2009) Psychological effects on interpersonal communication by bystander android using motions based on human- like needs. In: IEEE/RSJ intl conf on intelligent robots and systems, pp 3721-3726

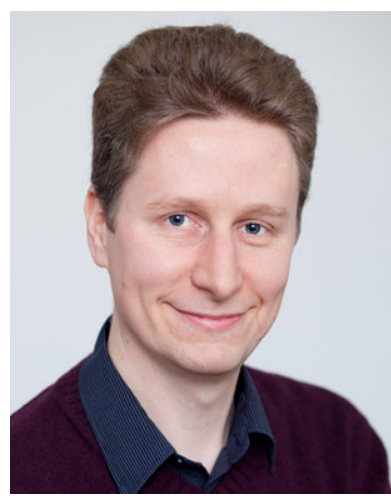

Christian Becker-Asano obtained his doctoral degree in 2008 from Bielefeld University. He then joined the Intelligent Robotics and Communication laboratories at ATR in Kyoto, Japan. Currently, he is Junior Fellow in the interdisciplinary research group at the Freiburg Institute for Advanced Studies. 